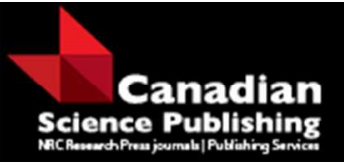

Canadian Journal of Forest Research

Revue canadienne de recherche forestière

\title{
Stochastic scheduling of single forest fire-fighting processor
}

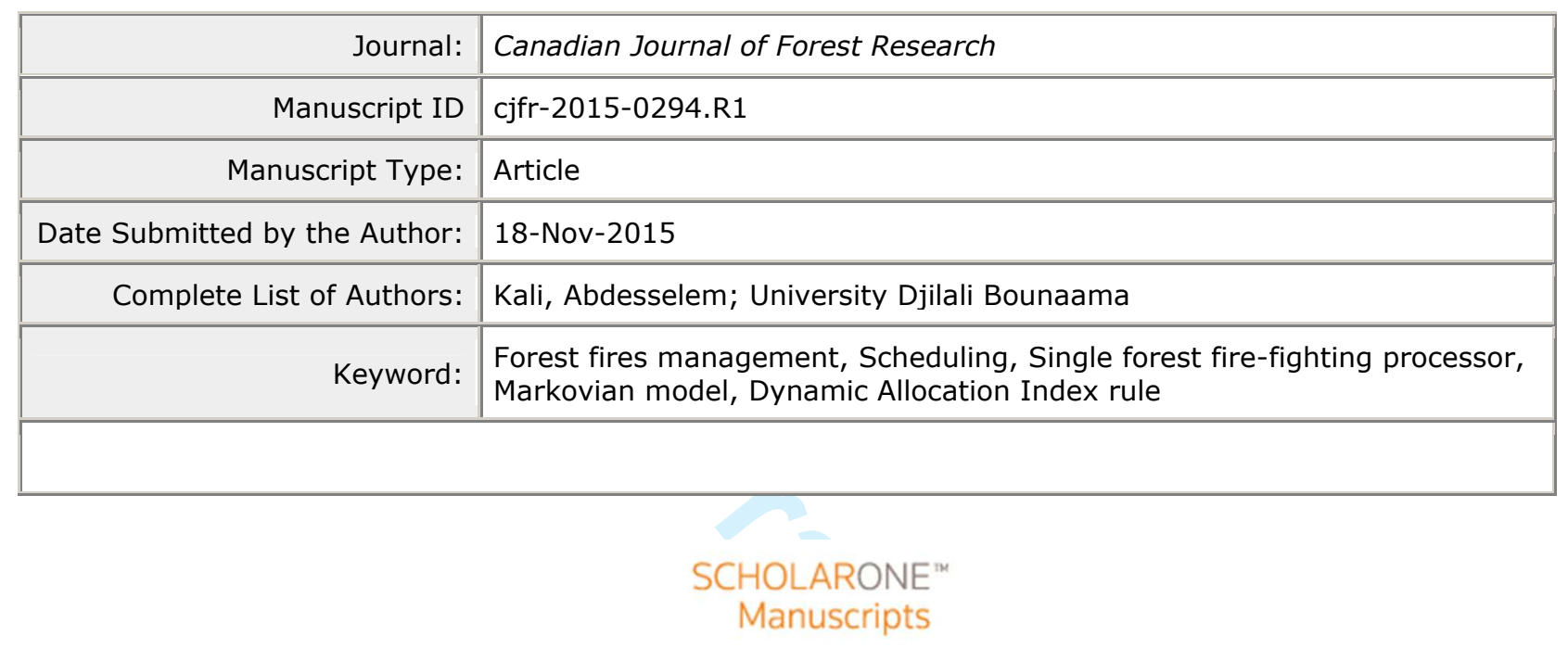


1 Full Title: Stochastic scheduling of single forest fire-fighting processor

2 Author: Abdesselem Kali

3 Institution: University Djilali Bounaama, Khemis-Miliana, Ain-Defla, Algeria

$4 \quad$ E-mail: abdesselem.kali@gmail.com

5 Telephone number: 00213555435606

6

7

8

9

10

11

12

13

14

15

16

17 
Abstract.

19 In forest fire-fighting, the longer the fires wait the larger they grow and the longer they take to

20 control. This study concerns the optimal deployment of single forest suppression processor of

21 initial attack in the case of fires ignited simultaneously. The aim is to minimize the total damage

22 caused by the fires to the burnt areas when all fires are suppressed. In "Scheduling fire-fighting

23 tasks using the concept of deteriorating jobs. Can. J. For. Res. 36: 652-658, (2006)", Rachaniotis

24 and Pappis use the concept of start-time dependent job processing-times for modelling the time

25 needed for fire suppression. The model is intricate but interesting in the sense that is based on

26 theoretical and empirical research in the field of forest fire-fighting. As a continuation, a

27 stochastic formulation which includes unpredicted parameters in the proposed model is

28 considered, and "Dynamic Allocation Index" rule is used to solve the problem. The optimality of

29 this rule and its effectiveness are proven. Experimental results depict the framework, inside it the

30 forest suppression processor achieves greater efficiency.

31 Keywords. Scheduling. Single forest fire-fighting processor. Markovian model. Dynamic

32 Allocation Index rule. 


\section{Résumé.}

40 Dans la lutte contre les feux de forêt, plus en tard plus les feux propagent et prennent du temps

41 pour leur confinement. Cette étude concerne le déploiement optimal d'un processeur de

42 suppression de la première intervention, dans le cas des incendies enflammés simultanément.

43 L'objectif est de minimiser les dommages dans les zones brûlées lorsque tous les feux sont

44 supprimés. Dans "Scheduling fire-fighting tasks using the concept of deteriorating jobs. Can. J.

45 For. Res. 36: 652-658, (2006)", Rachaniotis et Pappis montrent que la durée opératoire du

46 processeur dans l'extinction des incendies de forêt est dépendante du temps (détériorante). Le

47 modèle est difficile à utiliser mais intéressant dans le sens qu'il est basé sur la recherche

48 théorique et empirique dans le domaine de la lutte contre les feux de forêt. Dans cet article, une

49 formulation stochastique qui inclut les paramètres imprévus dans le modèle est considérée, et la

50 règle "d'Indice d'Allocation Dynamique" est utilisée pour résoudre le problème. L'optimalité de

51 cette règle et son efficacité ont fait leurs preuves. Les résultats expérimentaux déterminent le

52 cadre, à l'intérieur le processeur atteint une plus grande efficacité.

53 Mots-clés. Ordonnancement. Un seul processeur de la lutte contre les feux de forêt· Modèle

54 Markovien· Règle d'Indice d'Allocation Dynamique. 


\section{IntroducSection d'équation 1tion}

63 Fire is a natural component of many forest ecosystems but it also poses significant threats to 64 people and forest resources. The effectiveness of forest fire-fighting depends, in large part, on

65 the early detection of fire's ignition, on the accurate prediction of fire behaviour, and on the rapid

66 commitment of resources for intervention. The approaches to modelling forest fire have so far

67 involved mathematical analysis of physico-chemical phenomena, laboratory tests, and

68 observation of real cases of forest fire (Sullivan 2009a; Sullivan 2009b; Sullivan 2009c; Taylor

69 et al. 2013).

70 Scheduling, a field of Operational Research, supports decisions for preventive mobilization of

71 resources to contain fires in real time. A review of operations research contributions made in

72 forest fire management can be found in (Minas et al. 2012; Weintraub et al. 2007).

73 The problem of scheduling exists whenever there is a choice as to the order in which a number of

74 tasks can be performed (Conway et al. 1967). For our particular interest, the problem description

75 starts with a forest fire agency (a shop), and a set of fires (jobs). The forest fire agency is

76 completely described by giving the number and deferent types of fire-fighting resources of initial

77 attack (machines), namely: fire duty officers, trucks, fixed wing air tankers, helicopters, etc.

78 Fires can ignited either simultaneously or at different times. We can limit our attention to the

79 problem of simultaneously igniting fires which appears quite often in Mediterranean countries

80 (Vorisis 1999).

81 The relevant attributes of a fire that are given as part of the problem description are: processing-

82 time (the amount of time required to control the fire), release-time (the time at which fire 
83 suppression can be started) and deadline (the time at which fire can takes potentially destructive 84 behaviour and merit aggressive attack).

85 There are scheduling models in which single-machine is considered. From a practical point of 86 view, direct applications of this exception in forest fire-fighting are more frequent. Some fire-

87 fighting processors must operate separately or they can viewed as a single processor if they must 88 operate commonly.

89 Undeniably, the variability of fire processing-time as other attributes is inextricably involved.

90 There are various ways of regarding this variability. One can consider the processing-time of a

91 fire as a random variable or a fuzzy number unidentified in advance.

92 Based on the characteristics of the area where fire is ignited and on the characteristics of the

93 processor of initial attack, Rachaniotis and Pappis (2006) propose a model in which the

94 processing-time of a fire increases if the beginning of fire containment effort is delayed. This

95 fact is known in scheduling field as "deteriorating jobs".

96 With the objective to maximize the total value of the burnt areas remaining after the completion

97 of the containment operation, a branch and bound algorithm was presented in (Pappis and

98 Rachaniotis 2009). In the case where the objective is to minimize the total weighted tardiness, a

99 lower bound for the problem is given and a heuristic algorithm is used for the extraction of an

100 upper bound of the solution (Rachaniotis and Pappis 2011). Unfortunately, the proposed branch

101 and bound algorithms can yield optimal results for a moderate size of fires. The intricate

102 formulation of the model is among their basic deficiencies. In contrast, the deteriorating model

103 may help to give an insight and stimulation for future, more realistic approximations of real

104 forest management problems that are expected to be encountered (Rachaniotis and Pappis 2011). 
105 A stochastic approach that captures uncertainty and enables a more accurate description of the 106 decision making process may be adopted. Markov decision processes are a widely used tool to 107 model decision making under uncertainty. In the paper, a Markovian decision process for fire 108 processing-times evaluation based on the deteriorating model given in (Rachaniotis and Pappis 109 2006) is considered. The objective is the maximization of the total expected discounted value of

110 the burnt areas when all fires are suppressed. In an Markov decision process, the uncertainty in

111 transition probabilities may not be known precisely. In our case, this can happen because 112 quantities in the model of Pappis and Rachaniotis (2006) may have an inherent variability. The 113 probabilities might have been obtained via an estimation process. From the well-know "central 114 limit theorem" (Ross 1973), it is natural to consider the normal distribution for representing the 115 uncertainty in the transition probabilities. Dynamic Allocation Index rule (Gittins and Jones 116 1974) plays an important role in finding an optimal strategy for the Markovian process. Bellman 117 equations appear naturally in computing this priority rule. There are a number of methods to 118 solve the Bellman equation (See for example (Sonin 2006)). In our Markovian model, the 119 particular form of the transition matrix allows us to solve the corresponding Bellman equation 120 efficiency and analytically.

121 The remainder of this paper is composed of seven sections. We begin by giving a description of 122 the deterministic forest fire-fighting problem which can be modelled by deteriorating jobs.

123 Section 3 discusses considerations for stochastic approach. In Section 4, a Markovian model is 124 given for the stochastic scheduling problem when time proceeds in discrete steps. Dynamic 125 Allocation Index technique is used for determining the optimal non pre-emptive containment 126 policy. This result is given in Section 5. Section 6 provides the considered dynamic priority 
127 determining. Section 7 reports on our numerical tests. In Section 8, we conclude our work and 128 provide some recommendations for future development.

\section{2. A deteriorating model for scheduling some forest fire-fighting tasks}

130 In the scenario under consideration, $N$ forest fires are ignited simultaneously, detected and 131 reported, and one processor of initial attack is assigned for their containment.

132 All the $N$ fires evolve independently and no constraint is assumed which impose the order in 133 which fires must be suppressed.

134 The affected areas may require greater protection because of their characteristics: proximity of 135 population, social, economic, military, historic, etc.

136 As time elapses, the fire expands if there is a delay in the fire-fighting efforts.

137 The surviving value of the area at time $t$ where fire $F_{i}$ is ignited can be estimated by assigning a 138 discounted value $\alpha^{t} V_{i}$, where $V_{i}$ is a positive bounded constant and $\alpha$ lies strictly between 0 and

139 1. This estimate is done by taking into account all characteristics concerned the area.

140 The objective is to minimize the total damage caused by the fires to the burnt areas when all fires 141 are suppressed, that is the total discounted value of the surviving areas is maximized.

142 The processor is of limited transportation capacity $Q$ (in $L$ ) and application rate $\rho$ (in $L h^{-1}$ ), use 143 water as the means of fire suppression.

144 The processor is not required to extinguish a fire while busy suppressing another one, which 145 interpret that no pre-emption is allowed. 
146 During the containment of selected fire $F_{i}$, the processor can interrupt its operation as many

147 times as necessary, $\tau_{i}$ (in $h$ ) in total, for going to the nearest depository to refill and to come

148 back.

149 In general, square brackets [ ] will be used to denote position in sequence in a permutation

150 schedule. The symbol $F_{[i]}$ means the fire which is in the $i$ th position in suppression sequence.

151 Let $T_{0,[1]}$ (in $h$ ) be the time needed for the processor to travel from the base where it is stationed 152 to the spot where the first prioritized fire might be attacked.

$153 T_{[i],[i+1]}($ in $h), i=1,2, \ldots, N-1$, be the time required for the processor to travel from the front of 154 suppressed fire $F_{[i]}$ to the depository to refill and move to the front of fire $F_{[i+1]}$.

155 The times needed for the processor to travel for suppressing a prescribed fire or interruption for 156 refilling were considered as set-up times (as they would be in a typical manufacturing 157 environment).

158 For various forest fire-fighting considerations, the suppression of fire $F_{i}, 0 \leq i \leq N$, might be 159 started at time $r_{i}$. The release-time $r_{i}, r_{i}>0$, can simply represent the fire's initial inaccessibility 160 period. It represents, for example, the preparation time that precede the containment process.

161 The total forest area being burnt by fire $F_{[i]}$ at time $t$ at which fire's suppression effort starts, $162 E_{[i]}(t)$ (in $m^{2}$ ), as well as the relative amount of water needed to suppress the fire $F_{[i]}, \omega_{[i]}(t)$ (in $163 L \times m^{-2}$ ), deteriorates (increases) at a rate that depend upon the forest land that the fire burns, the 164 forest vegetation, and the weather conditions. 
$165 p_{[i]}(t)($ in $h)$ is the processing-time of fire $F_{[i]}$ if the "decision" of fire containment is taken at time

t. $p_{[i]}(t)$ deteriorates (increases) with $t$. The following expression holds (Rachaniotis and Pappis

167 2006):

168

$$
p_{[i]}(t)=\frac{E_{[i]}(t) \times \omega_{[i]}(t)}{\rho}+i n t\left(\frac{E_{[i]}(t) \times \omega_{[i]}(t)}{Q}\right) \times \tau_{[i]}
$$

169 where "int" is the integer part.

$170 E_{[i]}(t)$ is given in (Rachaniotis and Pappis 2006) by:

171

$$
E_{[i]}(t)=\frac{\pi}{\psi_{[i]}}\left(\frac{A_{[i]}}{B_{[i]}+1} \times t^{B_{[i]}^{+1}}+c_{[i]}(t) \times t\right)^{2}
$$

172 And $\omega_{[i]}(t)$ in (Katsanos 1978) is of the form:

173

$$
\omega_{[i]}(t)=1.2 \sqrt{A_{[i]} \times B_{[i]} \times t^{B_{[i]}-1}+c_{[i]}(t)}
$$

174 where $A_{[i]}$ and $B_{[i]}$ are constant coefficients depending on the varying environmental factors of

175 the fire in each area (wind speed, type of fuel and fuel loading) and/or the equilibrium rate of

176 spread (McAlpine and Wakimoto 1991).

$177 c_{[i]}(t)$ is a nonnegative coefficient at time $t$, defined as the difference between the forest fire's rate

178 of spread based on the fire behaviour prediction system and the fire rate of spread during the

179 fire's acceleration phase (McAlpine and Xanthopoulos 1998).

180 And $\psi_{[i]}$ is a constant depending on the wind speed $U_{[i]}\left(\right.$ in $\left.K m \times h^{-1}\right)$.

$181 \psi_{[i]}$ is given in (Rachaniotis and Pappis 2006) by the formula: 
$\psi_{[i]}=0.936 \times e^{0.07127 \times U_{[i]}}+0.461 \times e^{-0.043 \times U_{[i]}}$.

$184 \quad(4)$

$$
\begin{aligned}
& p_{[i]}(t)=\frac{1.2 \times \pi}{\psi_{[i]} \times \rho} \times\left(\frac{A_{[i]}}{B_{[i]}+1} \times t^{B_{[i]}+1}+c_{[i]}(t) \times t\right)^{2} \times \sqrt{A_{[i]} \times B_{[i]} \times t^{\left.B_{[i]}\right]^{-1}}+c_{[i]}(t)}+ \\
& \operatorname{int}\left(\frac{1.2 \times \pi}{\psi_{[i]} \times Q} \times\left(\frac{A_{[i]}}{B_{[i]}+1} \times t^{B_{[i]}+1}+c_{[i]}(t) \times t\right) \times \sqrt{A_{[i]} \times B_{[i]} \times t^{B_{[i]}-1}+c_{[i]}(t)}\right) \tau_{[i]}
\end{aligned}
$$

185 Let $C_{[i]}$ (in $h$ ) be the completion-time of $F_{[i]}$ that is the time at which $F_{[i]}$ 's suppression effort 186 stops. It is:

$$
C_{[i]}=\sum_{j=1}^{i} p_{[j]}\left(t_{[j]}\right)+\sum_{j=1}^{i}\left(T_{[j-1][[j]}+r_{[j]}\right)
$$

where $t_{[j]}$ is the decision-date for the containment of $F_{[j]}$, with $t_{[1]}=0$ and $t_{[j]}=C_{[j-1]}$ for $j=2,3$, $\ldots, N$, and $T_{[0],[1]}=T_{0,[1]}$

A fire $F_{[i]}$ has escaped initial attack if its containment time $C_{[i]}$ exceeds a specific "time limit" $d_{[i]}$

191 (in $h$ ), which can be considered as fire deadline.Section d'équation (suivante)

\section{3. Considerations for stochastic approach}

193 In the expression of $p_{[i]}(t)$ given by Equation (4), the exponent $B_{[i]}$ of $t$ is different in each fire 194 case. This fact makes the problem more complex and difficult to solve. In addition, whatever the 195 reliability of this model, it is important to realize that there is some, though very small, 196 probability that the unwanted and unexpected event will happen. This consideration is justified 
197 by the risks involved in a potential fire containment failure. Despite the research undertaken, the 198 statistical properties of a forest-fire are not fully known.

199 Fire safety design aims at providing solutions with risk levels that our society can tolerate. For a 200 diverse variety of types of insurance, normal distributions are extremely important in natural 201 sciences for real-valued random variables whose distributions are not known. It can be used to 202 maximize the chance of success for an acceptable level of risk or to minimize the risk for a 203 desired level of success.

204 Formally, the associated normal density $f_{[i]}(t)$ to fire $F_{[i]}$ is expressed as:

205

$$
f_{[i]}(t)=\frac{1}{\sigma \sqrt{2 \pi}} \exp \left(-\frac{(t-\mu)^{2}}{2 \sigma^{2}}\right)
$$

206 where $\mu$ and $\sigma$ are, respectively, the mean and standard deviation. $t$ is the completion-time of $F_{[i]}$ 207 given by (5).

208 The event: "The fire $F_{[i]}$ is controlled by time $d_{[i]}$ " must have a considerable probability of 209 occurrence. Consequently the mean $\mu$ might be $d_{[i]}$. The date $d_{[i]}$ cannot be considered as "hard 210 deadline" since wildfire often exceed this limit. A safety zone $\left[d_{[i]}, d_{[i]}+\varepsilon\right]$ must be considered in 211 which the probability of success in the suppression of $F_{[i]}$ has higher value. $\varepsilon$ depends naturally 212 on $s_{[i]}$ the starting-time of fire $F_{[i]}$ containment.

213 The value of the standard deviation $\sigma$ is related to the level of risk. If we take, for example, $5 \%$ 214 is the accepted risk then 2 standard deviations represent $d_{[i]}-r_{[i]}$. When the containment of fire 215 failure by time $d_{[i]}+\varepsilon$, the probability that the intended processor can suppress the fire decrease, 216 which is interpreted that the fire takes rage and merit additional fire-fighting efforts (See Figure 
217 1). The normal density $f_{i}(t)$ is used to estimate the probability of controlling the fire $F_{i}$ at any

218 time $t \geq s_{i}$. In Figure 1, the area $P_{1}$ under $f_{i}(t)$ between $s_{i}$ and $t_{1}$ represents the probability that $F_{i}$ is

219 controlled by time $t_{1}$ before $d_{i} . P_{1}$ increases with $t_{1}$ and when $s_{i}$ approaches $d_{i}$ the probability to

220 contain $F_{i}$ in $d_{i}$ decreases and safety zone $\left[d_{i}, d_{i}+\varepsilon\right]$ becomes wide. The probability that fire $F_{i}$ is

221 controlled in the safety zone must have higher values to that for $t_{1}=d_{i}$. This probability

222 decreases over time and coincides at time $t_{2}=d_{i}+\varepsilon$ with that at $t_{1}=d_{i}$. Exceeding $d_{i}+\varepsilon$, the fire is

223 uncontrollable and the probability to contain the fire decreases rapidly. These aforementioned

224 natural datum are justified by the expression taken for $P_{2}$. The value of $\varepsilon$ is then the solution of

225 the equation: $\int_{d_{i}}^{\varepsilon} f_{i}(t) d t=1-2 \int_{s_{i}}^{d_{i}} f_{i}(t) d t$.

226 Under the assumption of stationary probability transition matrix and total expected discounted

227 reward maximization criteria, the problem is a Markov decision problem (Bellmann 1956). In

228 theory, the problem can be solved by dynamic programming technique. However, the

229 combinatorial explosion of states puts severe limitations on finding optimal solutions. We solved

230 this problem optimally and efficiently by computing at each decision epoch a "priority" for each

231 unsuppressed fire, and selecting the fire which has the larger one.

232

\section{A Markovian model for the stochastic problem}

233 We denote by $S=\left\{\left(\Omega_{i}, \Gamma_{i}, P_{i}, V_{i}, \alpha\right), 1 \leq \mathrm{i} \leq N\right\}$ the associated cost-discounted Markov decision

234 process of the stochastic forest fire-fighting problem with the special features:

$235 \Omega_{i}$ is the state space of $F_{i}$, which may be in our case discrete.

236 Let $X_{i}(t)$ be the state of $F_{i}$ at time $t$, then the state of $S$ at time $t$ is $X(t)=\left(X_{1}(t), X_{2}(t), \ldots, X_{N}(t)\right)$. 
237 Fire $F_{i}$ enters some state of $\Omega_{i}$ denoted by the symbol $*_{i}$ if $F_{i}$ is completed. ${ }_{i}$ is the stationary

238 state of $F_{i}$ in which no transition to another state can be made.

239 At time $t$, the state $X_{i}(t)$ is then $*_{i}$ or it takes a nonnegative integer $\theta$, interpreted as the number of 240 time units already spent on $F_{i}$.

241 The action space $\Gamma_{i}$ consists of just two elements $a_{i}$ and $\overline{a_{i}}$. Action $a_{i}$ will be referred to as

242 "select $F_{i}$ for containment" or "maintain the suppression of $F_{i}$ if its treatment is started and is not

243 completed". In contrast, $\bar{a}_{i}$ will be referred to as "stop the suppression process of $F_{i}$ " off-course

244 if $F_{i}$ is extinguished.

245 If action $a_{i}$ is taken at time $t$, then the suppression of fire $F_{i}$ makes progress or completes during

246 the interval $[t, t+1$ [, and the state of all fires not in receipt of processor remain fixed to zero,

247 i.e., either $X_{i}(t+1)={ }^{*}$ or $X_{i}(t+1) \geq X_{i}(t)$

248 and $\forall j, j \neq i$ either $X_{j}(t+1)={ }_{j}$ or $X_{j}(t+1)=X_{j}(t)=0$.

249 Further, we shall only consider Markovian transitions. The transition probability from state $X_{i}(t)$

250 to state $X_{i}(t+1)$ is:

$$
\begin{aligned}
& P\left[X_{i}(t+1)={ }_{i} \mid X_{i}(t)={ }_{i}\right]=1, \\
& P\left[X_{i}(t+1)=*_{i} \mid X_{i}(t)=\theta, a_{i}\right]=\left\{\begin{array}{l}
\int_{s_{i}}^{\theta+1} f_{i}(t) d t \quad \text { if } s_{i} \leq \theta \leq d_{[i]} \\
1-\int_{s_{i}}^{\theta+1} f_{i}(t) d t \quad \text { if } \theta>d_{[i]}
\end{array},\right.
\end{aligned}
$$

252

253 (See Figure 1) and 


$$
P\left[X_{i}(t+1)=\theta+1 \mid X_{i}(t)=\theta, a_{i}\right]=1-P\left[X_{i}(t+1)=*_{i} \mid X_{i}(t)=\theta, a_{i}\right]
$$

255 with $X_{i}\left(s_{i}\right)=0$ and $f_{i}$ is the normal distribution given beforehand in (6).

256 In decision processes theory, a policy is defined as any rule for choosing at each decision epoch

257 that fire to be suppressed.

258 Remember that in Section 2, the optimal policy (if it exists) must to be non pre-emptive.

259 Decision times are then the initial time, and the fire completion-times.

260 Should action $a_{i}$ is taken at time $t$, an incremental rewards $\alpha^{t} V_{i}$ is earned. The current reward

$261 R(X(t))$ of the process $S$ is then $R(X(t))=\alpha^{t} V_{i}$

262 The objective function to be maximized is the total expected discounted reward function over an 263 infinite horizon:

$$
\mathrm{E}\left\{\sum_{t=0}^{+\infty} R(X(t)) \mid X(0)=0\right\},
$$

265 which can be written as:

$$
\mathrm{E}\left\{\sum_{i=1}^{N} \alpha^{t_{i}} V_{i}\right\}
$$

267 where $t_{i}$ is the first decision-time at which $F_{i}$ is selected for containment.

268 Note that (10) is well defined because rewards $V_{i}$ are bounded and $\left.\alpha \in\right] 0,1[$.

269 The general theory of discounted Markov decision process (Ross 1973) asserts the existence of 270 an optimal policy for the process $S$ which maximizes the total expected reward (10), which is 
271 deterministic, stationary, of Markov, and satisfies the optimality equations of dynamic

272 programming.

\section{5. Dynamic Allocation Index rule for optimal processing policy}

274 In this section, we denote by $k$ the last suppressed fire, and by $t$ its completion-time $C_{k}$. If no one 275 exists then $k=0$ and $t=0$.

276 If action $a_{i}$ is taken first at time $t$, and action $a_{j}$ is taken immediately after it, namely at time $t+$

$277 T_{k, i}+r_{i}+p_{i}(t)$, then the sum of discounted rewards obtained from the application of these two

278 actions is

$$
\alpha^{t} V_{i}+\alpha^{t+T_{k, i}+r_{i}+p_{i}(t)} V_{j}
$$

280 If the action order is interchanged, we obtain

$$
\alpha^{t} V_{j}+\alpha^{t+T_{k, j}+r_{j}+p_{j}(t)} V_{i}
$$

282 A little algebra shows that the first ordering has the greater reward if

$$
\frac{V_{i}}{1-\alpha^{T_{k, i}+r_{i}+p_{i}(t)}}>\frac{V_{j}}{1-\alpha^{T_{k, j}+r_{j}+p_{j}(t)}} .
$$

284 Using this idea, it is not hard to see that the total expected discounted reward obtained from the

$285 N$ fires is maximized by allocating at each decision epoch $t$ the processor to whichever

286 unsuppressed fire has the largest Dynamic Allocation Index $\gamma_{i}(t)$ computed as

$$
\gamma_{i}(t)=\frac{V_{i}}{1-\alpha^{T_{k, i}+r_{i}} \mathrm{E}\left\{\alpha^{p_{i}(t)}\right\}} .
$$


288 Let $D(t)$ denotes the set of delayed fires up to decision-time $t$. The following is the main result of 289 this paper.

290 Theorem 1. At each decision epoch $t$ it is optimal to choose action $a_{i}$ if and only if,

$291 \quad \gamma_{i}\left(X_{i}(t)\right)=\max _{j \in D(t)} \gamma_{j}\left(X_{j}(t)\right)=\max _{j \in D(t)} \frac{V_{j}}{1-\alpha^{T_{k, j}+r_{j}} \mathrm{E}\left\{\alpha^{p_{j}(t)}\right\}}$.

\section{6. Computing the Dynamic Allocation Index}

293 In this study, determining $\gamma_{i}\left(X_{i}(t)\right)$ passes by the calculation of

$$
\mathrm{E}\left\{\alpha^{p_{j}(t)}\right\}, j \in D(t)
$$

295 The simple form of the transition matrix of probabilities is the main virtue in computing (11).

296 Suppose that $\left(F_{[1]}, F_{[2]}, \ldots, F_{[i-1]}\right)$ is the current permutation of suppressed fires.

297 Then the current decision-time is $t=C_{[i-1]}$.

298 Denote by $M_{[i]}^{(0)}$ the probability generating function of $p_{[i]}(t)$ evaluated at $\alpha$ conditional upon

299 initial state " $X_{[i]}\left(s_{[i]}\right)=0$ ", where $s_{[i]}=C_{[i-1]}+T_{[i-1],[i]}+r_{[i]}$, i.e.

$$
M_{[i]}^{(0)}=\mathrm{E}\left\{\alpha^{p_{[i]}(t)} \mid X_{[i]}\left(s_{[i]}\right)=0\right\} .
$$

301 Using the geometric sum, it is easy to show that Equation (12) can be expressed as

302

$$
M_{[i]}^{(0)}=1-(1-\alpha) \mathrm{E}\left\{\sum_{h=0}^{p_{[i]}^{(t)-1}} \alpha^{h} \mid X_{[i]}\left(s_{[i]}\right)=0\right\} .
$$


303 Let $H_{[i]}^{(0)}$ denotes in Equation (13) the conditional expectation

304

$$
\mathrm{E}\left\{\sum_{h=0}^{p_{[i]}^{(t)-1}} \alpha^{h} \mid X_{[i]}\left(s_{[i]}\right)=0\right\}
$$

305

$H_{[i]}^{(0)}$ is obtained by solving the following Bellman equation (Ross 1973)

306

$$
H_{[i]}^{(y)}=1+\alpha \sum_{\left.h=y+1, \ldots, q-1,{ }^{*}{ }_{[i]}\right]} P_{[i]}^{(y, h)} H_{[i]}^{(h)}, \quad y=0,1, \ldots, q-1
$$

307

where in (14):

$$
\begin{aligned}
& q=p_{[i]}(t), \\
& H_{[i]}^{(q)}=0,
\end{aligned}
$$

308

$$
\begin{aligned}
& P_{[i]}^{(y, h)}=0, h>y+1, h \neq{ }_{[i]}^{*}, \\
& P_{[i]}^{(y, y+1)}=P\left[X_{[i]}\left(s_{[i]}+y+1\right)=y+1 \mid X_{[i]}\left(s_{[i]}+y\right)=y, a_{[i]}\right], y=0,1, \ldots, q-1, \\
& \left.P_{[i]}^{\left(y,{ }^{*}{ }^{i}\right]}\right)=P\left[X_{[i]}\left(s_{[i]}+y+1\right)={ }_{[i]} \mid X_{[i]}\left(s_{[i]}+y\right)=y, a_{[i]}\right], y=0,1, \ldots, q-1,
\end{aligned}
$$

$309 P$ is the transition probability defined in (7), (8) and (9).

310 Consequently,

$$
\begin{aligned}
& H_{[i]}^{(0)}=1+\alpha P_{[i]}^{(0,1)} H_{[i]}^{(1)}+\alpha P_{[i]}^{\left(0,{ }^{*}{ }_{[i]}\right)} H_{[i]}^{\left({ }_{[i]}\right)} \\
& \left.H_{[i]}^{(1)}=1+\alpha P_{[i]}^{(1,2)} H_{[i]}^{(2)}+\alpha P_{[i]}^{\left(1,{ }^{*}{ }^{*}\right)} H_{[i]}^{\left({ }^{*}\right]}\right)
\end{aligned}
$$

$311 \quad$ (15)

$$
\begin{aligned}
& \left.H_{[i]}^{(q-1)}=1+\alpha P_{[i]}^{(q-1, q)} H_{[i]}^{(q)}+\alpha P_{[i]}^{\left(q-1,{ }^{*}{ }^{*}\right)} H_{[i]}^{\left({ }^{*}{ }^{*}\right]}\right) \\
& H_{[i]}^{\left(*_{[i]}\right)}=1+\alpha P_{[i]}^{\left(*_{i i]}, *_{[i]}\right)} H_{[i]}^{\left(*_{[i]}\right)}
\end{aligned}
$$

312 The structure of system (15) provided us to solve it directly in ascent fashion. 
313 Namely,

314 (16)

$$
\begin{aligned}
& \left.H_{[i]}^{\left({ }^{*}\right]}\right)=\frac{1}{1-\alpha}, \\
& \left.H_{[i]}^{(q-1)}=1+\frac{\alpha}{1-\alpha} P_{[i]}^{\left(q-1,{ }^{*}[i]\right.}\right), \\
& \left.\left.H_{[i]}^{(0)}=1+\frac{\alpha}{1-\alpha} P_{[i]}^{\left(0,{ }^{*}{ }^{*}\right]}\right)+\sum_{j=1}^{q-1} \alpha^{j}\left(\prod_{l=0}^{j-1} P_{[i]}^{(l, l+1)}\right)\left(1+\frac{\alpha}{1-\alpha} P_{[i]}^{\left(j,{ }^{*}{ }^{*}\right)}\right)\right) .
\end{aligned}
$$

315 Consequently, the final form of the Dynamic Allocation Index of fire $F_{[i]}$ is then

316

$$
\gamma_{[i]}(t)=\frac{V_{[i]}}{1-\alpha^{T_{[i-1][i]}+r_{[i]}}(1-(1-\alpha)) H_{[i]}^{(0)}},
$$

317 where $H_{[i]}^{(0)}$ is given in (16), and $T_{[0],[1]}=T_{0,[1]}$.

\section{Numerical tests}

319 The reported Dynamic Allocation Index, given by Equation (17), was coded and run in Scilab-

$321 \mathrm{GHz}$ and 4 Go of RAM Under Windows 7 (64 bits). All parameters are randomly generated

322 according to the uniform law in specific intervals esteemed using experimental data from

323 (McAlpine and Wakimoto 1991) (see (Rachaniotis and Pappis 2006)):

$\left.324 A_{i} \in\right] 0,14\left[\right.$ and $\left.B_{i} \in\right] 1,2[$.

325 In general $\left.c_{i}(t) \in\right] 0,11\left[\right.$ for $U_{i} \in\{0,1, \ldots, 8\}$.

$326 \alpha \in] 10^{-6}, 10^{-4}\left[\right.$ and $\left.V_{i} \in\right] 10^{5}, 2 \times 10^{5}[$.

$327 \rho=6.48 \times 10^{6}\left(\right.$ in $\left.L h^{-1}\right)$ and $Q=3 \times 10^{4}(L)$ (estimations). 
328 In this experimentation, it is assumed that $\tau_{i}=0.2$ (in $\left.h\right), T_{0,[1]}$ and $T_{[i],[i+1]}$ are in $] 0,1[$ (in $h$ ).

329 The standard deviation $\sigma_{i}$ is taken randomly according to the uniform law, as envisaged for the

330 other parameters in this experimentation, in the set $\left\{\frac{d_{i}-r_{i}}{4}, \frac{d_{i}-r_{i}}{3}, \frac{d_{i}-r_{i}}{2}, d_{i}-r_{i}\right\}$, since

331 almost all of the area under the bell curve lies within 4 standard deviations from the mean. Thus,

332 outliers more than 4 standard deviations from the mean will be extremely rare.

333 In our tests, we mainly interested in estimating the deadline $d$ of fires containment and, in respect

334 of this delay, the number of fires that can be surmounted by the processor. For this purpose, $d$

335 taken integer was raised by "one" from 1 to 50 (in $h$ ). For each $d$ value, the algorithm has been

336 applied on a variety of instances (1000 instances in total, each of which contains 100 cases of

337 fires).

338 To get accuracy in the calculation of the Dynamic Allocation Indices, we set the number of fire's

339 states to 100 states.

340 For each value of $d$ and for the 1000 instances generated for this delay, we tabulate the average

341 of numbers of fires suppressed by the processor (denoted $\tilde{N}$ ), the average of the containment

342 times for the first fires selected by the index rule and escaped the efforts of initial attack (denoted

$343 \hat{C}$ ), and the average of CPU time consumption (in sec) (denoted $\check{T}$ ). These results are reported in

344 Table 1. It appears that only 07 fires (on average) can be confined within the period of 11 to 50

345 first hours (on average). With $d$ between 11 and 50, the time required to control the 8th fire

346 deteriorates and exceeds all barriers feasible in practice. The calculation time for each instance is

347 about 1.6 second.

\section{8. Conclusion}


349 Regardless of the complex structure of the model by Rachaniotis and Pappis (2006), and of 350 inability to solve the problem perfectly, it may be also argued that uncertainty can enter the 351 model because quantities in the model have an inherent variability. We have addressed a novel 352 approach for the optimal solution when uncertainty occurs. A Markovian decision process is 353 developed, and Dynamic Allocation Index technique is used to solve the problem. The optimality 354 of this rule is proved, and its determination is also explained. By field tests, fires deadline are at 355 most 11 hours, and only 07 fires can be surmounted by the processor.

356 In our Markovian process the rewards are fixed, so that the only parameters that vary are the 357 transition probabilities. To our knowledge, the statistical properties of forest-fire are not fully 358 known. We adopt normal distribution for representing the uncertainty in the transition 359 probabilities. The normal distribution is most often assumed to describe the random variation 360 that occurs in the data from many scientific disciplines with condition that their distributions 361 should be unknown. The choice of the mean to be the deadline of fire and standard deviation 362 governed by the risk of fire suppression failure seem natural in forest fires-fighting.

363 A notable direction of study is to consider the problem of scheduling a single fire-fighting 364 processor under incomplete information about the normal distribution means as in (Katehakis 365 and Robbins 1995), or incomplete information about the fire containment lengths as in (Burnetas 366 and Katehakis 1993), or use the consistent estimates of the transition law and reward as defined 367 in (Burnetas and Katehakis 1997).

368 In our study, fires are ignited at the same time. The generalization to the case of fires ignited at 369 different times, as depicted in (Rachaniotis and Pappis 2006), is quite simple. Thereby, dispute 370 the difficulty of Poissonian arrivals (Blake 1979) seems worthwhile. It is also interesting to 371 discuss the case in which fires are disadvantaged during their containment. In this situation, and 
372 unlike the deteriorating models, the suppression of a fire generates a supplementary processing373 time to complete the fire.

374 The problem of scheduling parallel identical or non-identical fire-fighting processors is treated 375 by Pappis and Rachaniotis (2010) as a job-scheduling with deteriorating jobs. In this problem the 376 pre-emption is allowed with a variable number of processors used by a fire over time. An 377 interesting model for fire processing-times is formulated depending on the number and type of 378 each processor employed to contain a fire. A stochastic continuation of this model is almost 379 similar to that developed in Section 4 and the dynamic allocation index $\gamma_{i}$ can be generalized 380 without jeopardizing the optimality of the index rule. With each fire $F_{i}$, which is at time $t$ in state $381 X_{i}(t)$, is associated a priority $\gamma_{i}\left(X_{i}(t)\right)=\sup _{\xi>t} \frac{V_{i}}{1-\alpha^{T_{i}+r_{I}} \mathrm{E}\left\{\alpha^{\xi}\right\}}$

382 where $T_{i}$ is the time required to move the needed processors from other sites to the spot where 383 fire $F_{i}$ is ignited, and $\xi$ is the stopping-time of $F_{i}$. $\xi$ is a decision variable that depends on the fire 384 processing-time calculated at time $t$. This prioritization allows the decision-maker to allocate a 385 minimal set of required processors to the fire with the most highly index. In a case of a tie, any 386 well known rule is employed to break it. An important property of Dynamic Allocation Index $\gamma_{i}$ 387 is that the supremum is achieved by $\xi=\min _{h>t}\left\{h, \gamma_{i}\left(X_{i}(h)\right)<\gamma_{i}\left(X_{i}(t)\right)\right\}$ and by any stopping time $388 \sigma>t$ which satisfies $\sigma \leq \xi$ and $\gamma_{i}\left(X_{i}(\sigma)\right) \leq \gamma_{i}\left(X_{i}(t)\right)$. At each decision epoch, which corresponds to 389 an optimal stopping-time or a completion-time or new arrival of a fire, the processing-times of uncompleted fires are recalculated for new indexation and new assignments of resources are 
392 In Pappis and Rachaniotis (2010), "when there are more processors available than those already

393 assigned, which are simultaneously required by the fires already ignited, a maximal possible

394 number of processors are assigned. Otherwise, processors are shared by fires with equal ratios so

395 that their heights decrease at the same rate. The processors are assigned according to non

396 ascending order of the ratios containment rate/travelling time, which depends directly on the

397 distance from their location to the fire". Please note that the action taken at each decision epoch $t$

398 is always optimal with regard to the state resulting from the allocation of residual processors to

399 fires already ignited up to time $t$.

\section{References}

401 Bellmann, R.E. 1956. A Problem in the Sequential Design of Experiments. Sankhya. 16: 221$402 \quad 229$.

403 Blake, IF. 1979. An Introduction to Applied Probability. John Wiley and Sons, New York.

404 Burnetas, A.N. and Katehakis, M.N. 1993. On Sequencing Two Types of Tasks on a Single

405 Processor under Incomplete Information. Probability in the Engineering and Informational

406 Sciences. 7 (1): 85-0119.

407 Burnetas, A.N. and Katehakis M.N. 1997. Optimal Adaptive Policies for Markov Decision

408 Processes. Mathematics of Operations Research. 22 (1): 222-255.

409 Conway, R.W., Maxwell, W.L., and Miller, L.W. 1967. Theory of scheduling. Addison-Wesley, 410 Reading, Mass. 
411 Gittins, J.C., and Jones, D.M. 1974. A Dynamic Allocation Index for the Sequential Design of 412 Experiments. Proc. of European Meeting of Statisticians 1972 and in Progress in Statistics, ed. J. 413 Gani, North Holland. 241-266.

414 Katehakis, M.N., and Veinott Jr., A.F. 1987. The Multi-Armed Bandit problem: decomposition 415 and computation. Mathematics of Operations Research. 22 (2): 262-268.

416 Katehakis, M.N., and Robbins H.E. 1995. Sequential choice from several populations.

417 Proceedings of the National Academy of Sciences U.S.A. 92: 8584-8565.

418 Katsanos, A. 1978. Suppression of forest fires by air. Ministry of Agriculture, Department of 419 Forest Research, Athens, Greece. 40: 13-73.

420 McAlpine, R.S., and Wakimoto, R.H. 1991. The acceleration of fire from point source to 421 equilibrium spread. For.Sci. 37(5):1314-1317.

422 McAlpine, R.S., and Xanthopoulos, G. 1998. Predicted vs. observed fire spread rates in 423 ponderosa pine fuel beds: a test of American and Canadian systems. In Proceedings of the 10th 424 Conference on Fire and Forest Meteorology, Ottawa, Ont., 17-21 April 1989. Forestry Canada, 425 Ottawa, Ont. 287-294.

426 Minas, J.P., Hearne, J.W., and Handmer, J.W. 2012. A review of operations research (OR) 427 methods applicable to wildfire management. International Journal of Wildland Fire. 21(3): 189428196.

429 Pappis C.P., and Rachaniotis, N.P. 2009. Scheduling a single fire fighting resource with 430 deteriorating fire suppression times and set-up times. Oper. Res. Int. J. doi:10.1007/s12351 -009$431 \quad 0038-6$. 
432 Pappis C.P., and Rachaniotis, N.P. 2010. Scheduling in a multi-processor environment with

433 deteriorating job processing times and decreasing values: the case of forest fires. Journal

434 of Heuristics $16(4), 617-632$.

435 Rachaniotis, N.P., and Pappis, C.P. 2006. Scheduling fire-fighting tasks using the concept of 436 deteriorating jobs. Can. J. For. Res. 36: 652-658.

437 Rachaniotis, N.P., and Pappis, C.P. 2011. Minimizing the total weighted tardiness in wildfire 438 suppression. Operational Research. 11(1): 113-120.

439 Ross, S.M. 1973. Applied probability models with optimization applications. Holden-Day, San

$440 \quad$ Francisco.

441 Sonin, I. 2006. The Optimal Stopping of Markov Chain and Recursive Solution of Poisson and

442 Bellman Equations. From Stochastic Calculus to Mathematical Finance. 609-621.

443 Sullivan, A.L. 2009a. Wildland surface fire spread modelling, 1990-2007. 1: Physical and quasi-

444 physical models. International Journal of Wildland Fire. $18: 349-368$.

445 Sullivan, A.L. 2009b. Wildland surface fire spread modelling, 1990-2007. 2: Empirical and 446 quasi-empirical models. International Journal of Wildland Fire. $18:$ 369-386.

447 Sullivan, A.L. 2009c. Wildland surface fire spread modelling, 1990-2007. 3: Simulation and 448 mathematical analogue models. International Journal of Wildland Fire. 18: 387-403.

449 Taylor, S.W., Woolford, D.G., Dean, C.B., and Martell, D.L. 2013. Wildfire Prediction to Inform 450 Fire Management: Statistical Science Challenges. Statistical Science. 28(4) : 586-615. 
451 Vorisis, D. 1999. Definition of the needs of suppress groups as concern of the prediction of the

452 behaviour of the wild land fires. International Symposium, Forest Fires: Needs and Innovations,

453 EC, DG XII, Athens, Greece. 18-19.

454 Weintraub, A., Romero, C., Bjфrndal, T., Epstein, R., and Miranda, J. 2007. Handbook of

455 operations research in natural resources (International series in operations research and

456 management science), Springer.

457

458

459

460

461

462

463

464

465

466

467

468

469 
Table 1. Experimentation results

471

472

473

474

475

476

477

478

479

\begin{tabular}{llllllllllll}
\hline$d$ & $\tilde{N}$ & $\hat{C}$ & $\check{T}$ & $d$ & $\tilde{N}$ & $\hat{C}$ & $\check{T}$ & $d$ & $\tilde{N}$ & $\hat{C}$ & $\check{T}$ \\
\hline $\mathbf{1}$ & 1.22 & 1.37 & 1.03 & $\mathbf{1 8}$ & 7.14 & 537.2 & 1.56 & $\mathbf{3 5}$ & 7.76 & 30272. & 1.61
\end{tabular}

$\begin{array}{lll}2 & 2.54 & 2.37\end{array}$

$1.18 \quad 19$

$197.06 \quad 839.6$

1.57

367.6

$3 \quad 3.86 \quad 3.69$

$\begin{array}{llll}1.31 & 20 & 7.34 & 4260 .\end{array}$

1.58

$\begin{array}{llll}37 & 7.14 & 41890 . & 1.59\end{array}$

$\begin{array}{lll}4 & 5.18 & 6.47\end{array}$

$1.42 \quad 21$

$5 \quad 5.86 \quad 11.40$

1.48

217.08

12791. 1.57

$\begin{array}{llll}38 & 7.36 & 12334 . & 1.58\end{array}$

$\begin{array}{lll}6 & 6.24 & 22.95\end{array}$

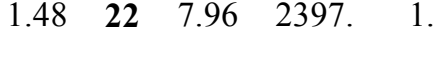

$\begin{array}{llll}39 & 7.8 & 45222 . & 1.60\end{array}$

$7 \quad 6.3$

$6.3 \quad 20.96$

$1.49 \quad 23$

3.245620.

1.6

$\begin{array}{llll}40 & 7.62 & 108125 . & 1.59\end{array}$

86

$\begin{array}{lllll}1.52 & 24 & 7.1 & 5000 . & 1.59\end{array}$

$41 \quad 7.18 \quad 564072 . \quad 1.59$

$\begin{array}{llllllll}\mathbf{9} & 6.8 & 120.53 & 1.53 & \mathbf{2 6} & 7.7 & 14101 . & 1.59\end{array}$

$427.36 \quad 12334 . \quad 1.58$

480

$\begin{array}{llllllllllll}10 & 6.72 & 196.28 & 1.53 & \mathbf{2 7} & 7.5 & 10661 . & 1.6 & \mathbf{4 4} & 7.66 & 108284 & 1.62\end{array}$

481

$\begin{array}{llllllllllll}11 & 7.08 & 98.76 & 1.54 & \mathbf{2 8} & 7.1 & 10352 . & 1.59 & \mathbf{4 5} & 7.18 & 564072 . & 1.61\end{array}$

$\begin{array}{llllllllllll}12 & 7.14 & 156.5 & 1.54 & \mathbf{2 9} & 7.24 & 2801 . & 1.6 & \mathbf{4 6} & 7.88 & 186788 . & 1.62\end{array}$

482

$\begin{array}{llllllllllll}13 & 7.08 & 164.48 & 1.54 & \mathbf{3 0} & 7.7 & 14101 . & 1.59 & \mathbf{4 7} & 7.68 & 108302 . & 1.62\end{array}$

483

$\begin{array}{llllllllllll}14 & 7.06 & 403.24 & 1.55 & \mathbf{3 1} & 7.56 & 11212 . & 1.61 & \mathbf{4 8} & 7.2 & 582567 . & 1.662\end{array}$

$\begin{array}{llllllllllll}15 & 7.18 & 1721.6 & 1.56 & \mathbf{3 2} & 7.12 & 10577 . & 1.6 & \mathbf{4 9} & 7.38 & 13026 . & 1.62\end{array}$

484

$\begin{array}{llllllllllll}16 & 7.18 & 486.37 & 1.56 & \mathbf{3 3} & 7.32 & 10197 . & 1.589 & \mathbf{5 0} & 7.92 & 332857 . & 1.634\end{array}$

485

$\begin{array}{llllllll}17 & 7.2 & 449.96 & 1.57 & \mathbf{3 4} & 7.66 & 18542 . & 1.58\end{array}$

486

487

488

489

490

491

492

493

494 
Fig.1. The probability determining: $P_{1}$ and $P_{2}$ are respectively the probabilities that fire $F_{i}$ is controlled at time $t_{1}$ before time $d$ and at time $t_{2}$ after time $d$.

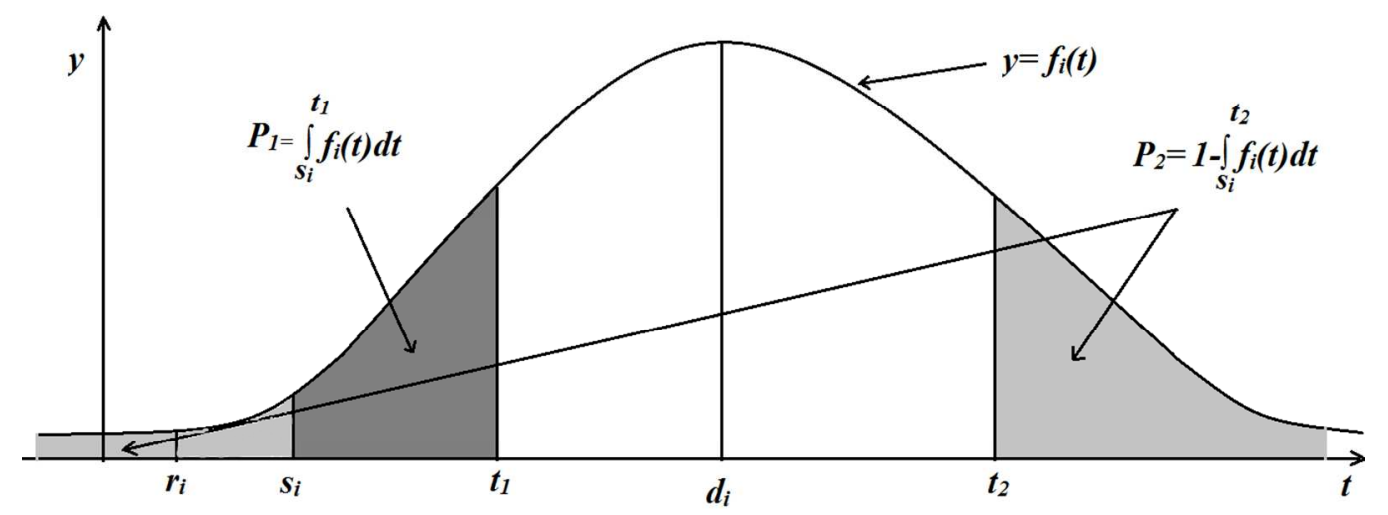




\section{$\underline{\text { Scilab Source Code }}$}

2 1. Variables and parameters definition

3 2. File 1: DAI.sce (Main File)

4 3. File 2: Data.sci (Data of the problem)

5 4. File 3: Schedule.sci (A schedule of selected fires)

6 5. File 4: processing_time.sci (Processing time of a selected fire)

7 6. File 5: starting_time.sci ( fire starting-time)

8 7. File 6: completion_time.sci (Completion time of a selected fire)

9 8. File 7: cdfnormal.sci (cumulative distribution function of normal distribution, Written by Philippe.CASTAGLIOLA@, univ-nantes.fr Université de Nantes \& IRCCyN UMR 10.File 9: DAI_fire.sci (Dynamic Allocation Index of fire) 


\section{1. Variables and parameters definition}

26 NS : Number of states

$27 \quad$ NI : Number of instances

28 NF : Number of fires

29 SF : Set of fires

30 A and $\mathbf{B}$ : Constant coefficients depending on the varying environmental factors of the fire in 31 each area (wind speed, type of fuel and fuel loading) and/or the equilibrium rate of spread

$32 \mathbf{U}$ : Wind speed in the area where the fire is ignited

c : Nonnegative coefficient, defined as the difference between the forest fire's rate of spread

34 based on the fire behaviour prediction system and the fire rate of spread during the fire's

35 acceleration phase

Gamma : constant depending on the wind speed $\mathbf{U}$

37 alpha : a fixed discount factor

$38 \quad \mathbf{V}$ : the associated bounded value to the area where fire is ignited

ro: application rate of the processor

$\mathbf{Q}$ : transportation capacity of the processor

thau : the necessary time for the processor for going to the nearest depository of the area

42 where fire is ignited to refill and to come back

$43 \mathbf{T}(\mathbf{0 , f})$ : the time needed for the processor to travel from the base where it is stationed to the $44 \quad$ spot where the fire $f$ might be attacked 
$45 \mathbf{T}(\mathbf{f} 1, \mathbf{f} 2)$ : is the time required for the processor to travel from the front of suppressed fire $\mathbf{f 1}$ to

46 the depository to refill and move to the front of fire $\mathbf{f} 2$

$47 \quad \mathbf{r}$ : release-date of fire

48 cdfnormal: cumulative distribution function of normal distribution

49 f : fire

50 C : completion-time

$51 \mathbf{s}$ : starting-time

52 p : processing-time

53 DAI: Dynamic Allocation Index of a fire

$54 \quad$ TM : transition matrix

55 step : step

56 d: deadline

57 D : deviation

$58 \quad$ Pr : transition probability

$59 \quad$ LSF : last selected fire

60 CTLSF : completion of last selected fire

61 SCHEDULE : a sequence of suppressed fires

62 Tr_Mat : is a matrix, contains all fire's Transition Matrices of probabilities

63 pos : position in the schedule 


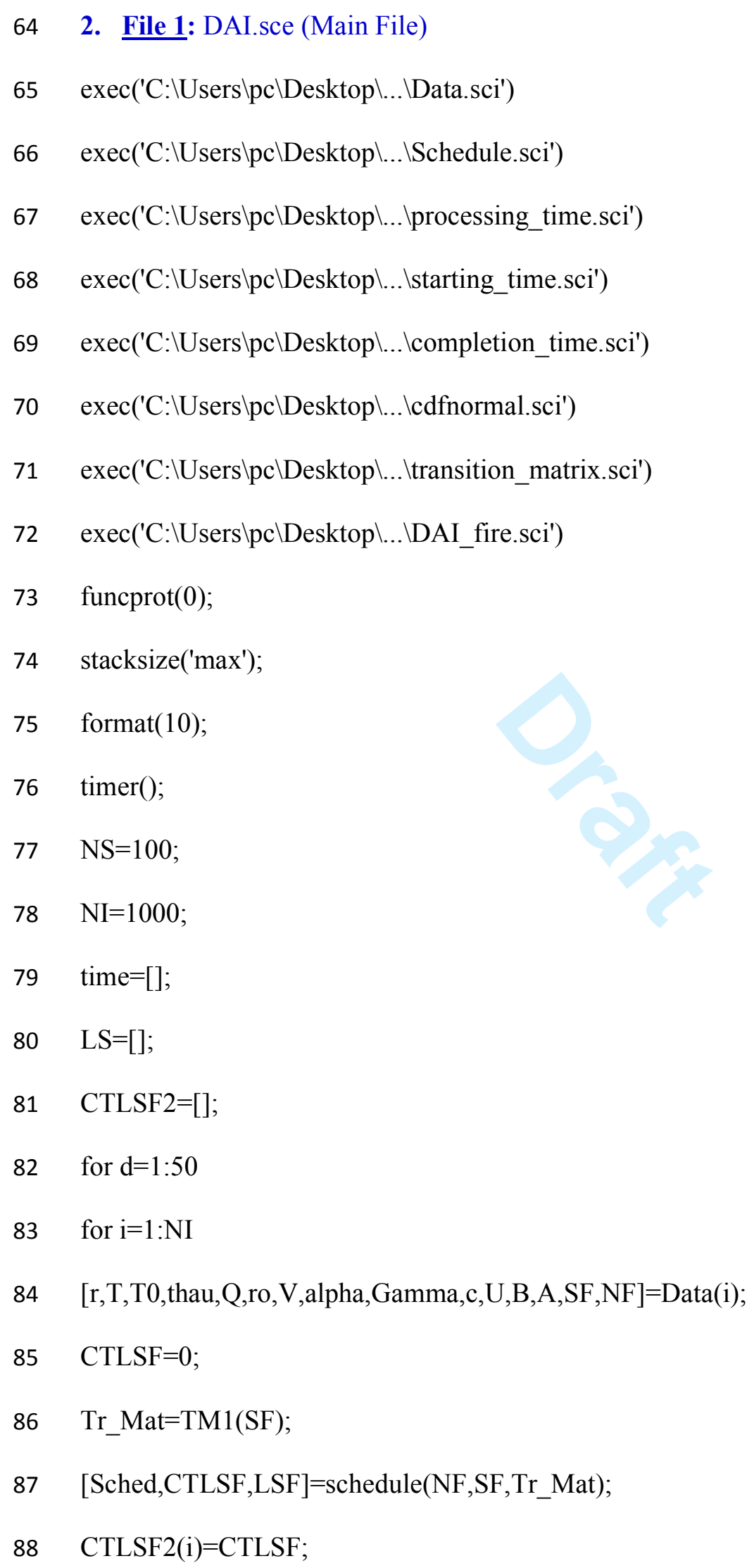


89 LS(i)=length(Sched);

90 time(i)=timer();

91 end

92 average_running_time $(\mathrm{d})=\mathrm{sum}($ time$) / \mathrm{NI}$;

93 average_Lenght_Schedule $(\mathrm{d})=\operatorname{sum}(\mathrm{LS}) / \mathrm{NI}$;

94 average_completion_time $(\mathrm{d})=\mathrm{sum}(\mathrm{CTLSF} 2) / \mathrm{NI}$;

95 end

96 average_running_time

97 average_Lenght_Schedule

98 average_completion_time

99

100

101

102

103

104

105

106

107

108

109 
110 3. File 2: Data.sci (Data of the problem)

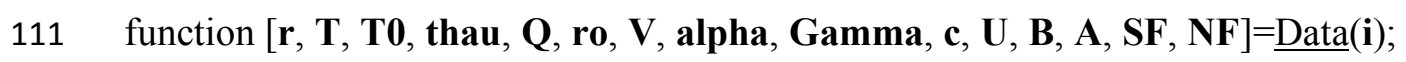

$112 \quad \mathbf{N F}=\operatorname{grand}(1,1$, "uin", 100,100)

$113 \mathbf{S F}=[1: \mathbf{N F}]$

$114 \quad \mathbf{A}=\operatorname{grand}(1, \mathbf{N F}$, "unf",0,14);

115 B=grand(1,NF,"unf",1,2);

$116 \mathbf{U}=$ grand(1,1,"uin",0,8);

$117 \mathbf{c}=\operatorname{grand}(1, \mathbf{N F}$, "unf",0,11);

$118 \mathbf{G a m m a}=0.936 * \exp (0.07127 * \mathbf{U})+0.461 * \exp (-0.043 * \mathbf{U})$;

119 alpha= $\operatorname{grand}(1,1$, "unf",0.000001,0.0001);

120 V=grand(1,NF,"unf", 100000,200000);

$121 \quad$ ro $=6480000$

$122 \quad \mathbf{Q}=30000$;

123 thau $=0.2$;

124 T0= grand(1,NF,"unf",0,1);

125 for $\mathrm{j}=1: 1:(\mathbf{N F}-1)$

126 for $\mathbf{i}=\mathbf{j}+1: 1: \mathbf{N F}$

$127 \mathbf{T}(\mathrm{j}, \mathbf{i})=\operatorname{grand}\left(1,1\right.$, "unf $\left.^{\prime \prime}, 0,1\right)$;

$128 \quad \mathbf{T}(\mathbf{i}, \mathrm{j})=\mathbf{T}(\mathrm{j}, \mathbf{i})$;

129 end

130 end

$131 \quad \mathbf{r}=\operatorname{grand}(1, \mathbf{N F}, " u n f ", 0,1)$;

132 endfunction 
135 4. File 3: Schedule.sci (A schedule of selected fires)

136 function $[$ Sched, CTLSF, LSF $]=\underline{\text { schedule }}\left(\mathbf{N F}, \mathbf{S F}, \mathbf{T r} \_\right.$Mat $)$

137 for $p o s=1: \mathbf{N F}$

$138 \mathrm{t}=0$;

139 indices $=[]$;

140 for $\mathrm{f}=\mathbf{S F}$

141 TM=Tr_Mat(:,1+t:NS+1+t);

142 indices $=[$ indices, $\mathrm{DAI}(\mathrm{TM})]$

$143 \mathrm{t}=\mathrm{NS}+1+\mathrm{t}$

144 end

145 max_DAI=max(indices);

$146 \mathrm{i}=$ find(indices $==$ max_DAI);

$147 \quad \mathbf{L S F}=\mathbf{S F}(\mathrm{i})$

$148 \quad \mathbf{C T L S F}=\mathrm{C}(\mathbf{S F}(\mathrm{i}))$;

149 if CTLSF $>$ d then

150 break

151 else

$152 \operatorname{Sched}($ pos $)=$ SF $(i)$;

$153 \quad \mathbf{S F}(\mathrm{i})=[]$

154 Tr_Mat $\left(:,(\mathrm{i}-1) *(\mathrm{NS}+1)+1: i^{*}(\mathrm{NS}+1)\right)=[]$;

155 end;

156 end

157 endfunction

158

159 
160 5. File 4: processing_time.sci (Processing time of a selected fire)

161 function $\mathbf{y}=\underline{p}(\mathbf{f})$

$162 \mathbf{y}=((1.2 * \% \text { pi }) /(\text { Gamma*ro }))^{*}\left(\left(\left(\left(\mathrm{~A}(\mathbf{f}) *\left(\mathrm{~s}(\mathbf{f})^{\wedge}(\mathrm{B}(\mathbf{f})+1)\right)\right) /(\mathrm{B}(\mathbf{f})+1)\right)+\mathrm{c}(\mathbf{f}) * \mathrm{~s}(\mathbf{f})\right)^{\wedge} 2\right)^{*} \operatorname{sqrt}\left(\mathrm{A}(\mathbf{f}) * \mathrm{~B}(\mathbf{f})^{*}\right.$

$\left.163\left(\mathrm{~s}(\mathbf{f})^{\wedge}(\mathrm{B}(\mathbf{f})-1)\right)+\mathrm{c}(\mathbf{f})\right)+\operatorname{int}\left(((1.2 * 0 \% \mathrm{pi}) /(\mathrm{Gamma} * \mathrm{Q}))^{*}\left(\left(\left(\left(\mathrm{~A}(\mathbf{f})^{*}\left(\mathrm{~s}(\mathbf{f})^{\wedge}(\mathrm{B}(\mathbf{f})+1)\right)\right) /(\mathrm{B}(\mathbf{f})+1)\right)+\right.\right.\right.$

$\left.\left.\left.164 \mathrm{c}(\mathbf{f})^{*} \mathrm{~s}(\mathbf{f})\right)^{\wedge} 2\right)^{*} \operatorname{sqrt}\left(\mathrm{A}(\mathbf{f})^{*} \mathrm{~B}(\mathbf{f})^{*}\left(\mathrm{~s}(\mathbf{f})^{\wedge}(\mathrm{B}(\mathbf{f})-1)\right)+\mathrm{c}(\mathbf{f})\right)\right)^{*}$ thau

165 endfunction

166

167

168

169

170

171

172

173

174

175

176

177

178

179

180

181

182

183

184 
185 6. File 5: starting_time.sci (fire starting-time)

186 function $\mathbf{y}=\underline{s}(\mathbf{f})$

187 if $\mathrm{CTLSF}==0$ then

$188 \quad \mathbf{y}=\mathrm{T} 0(\mathbf{f})+\mathrm{r}(\mathbf{f})$

189 else

$190 \mathbf{y}=\mathrm{CTLSF}+\mathrm{T}(\mathrm{LSF}, \mathbf{f})+\mathrm{r}(\mathbf{f})$

191 end

192 endfunction

193

194

195

196

197

198

199

200

201

202

203

204

205

206

207

208

209 
210 7. File 6: completion_time.sci (Completion time of a selected fire)

211 function $\mathbf{y}=\underline{C}(\mathbf{f})$

$212 \quad \mathbf{y}=\mathrm{s}(\mathbf{f})+\mathrm{p}(\mathbf{f})$

213 endfunction

214

215

216

217

218

219

220

221

222

223

224

225

226

227

228

229

230

231

232

233

234

10

https://mc06.manuscriptcentral.com/cjfr-pubs 
235

236

237

238

239

240

241 error("incorrect number of arguments")

242 end

243 if exists("mu","local")

$244 \mathbf{m u}=0$

245 end

246

$247 \quad \operatorname{sigma}=1$

248 end

249 if $\operatorname{sigma}<=0$

250 error("argument "sigma" must be $>0$ ")

251 end

25

253

254

255

256

257

258

259 
260 9. File 8: transition_matrix.sci (Matrix of transition probabilities)

261 function $\mathbf{y}=\underline{\mathrm{TM}}(\mathbf{f})$

262 step $=\underline{\text { linspace }}(\mathrm{s}(\mathbf{f}), \mathrm{C}(\mathbf{f}), \mathrm{NS})$

$263 \operatorname{step}(1)=[]$

$264 \mathrm{n}=\operatorname{grand}(1,1$, uin", 1,4)

$265 \mathrm{D}=(\mathrm{d}-\mathrm{r}(\mathbf{f})) / \mathrm{n}$

$266 \mathrm{k}=1$

267 for $\mathrm{t}=$ step

268 if $\mathrm{t}<=\mathrm{d}$ then

$269 \operatorname{Pr}(\mathrm{k}, \mathrm{k}+1)=$ cdfnormal( $\mathrm{t}, \mathrm{d}, \mathrm{D})-\operatorname{cdfnormal}(\mathrm{s}(\mathbf{f}), \mathrm{d}, \mathrm{D})$

$270 \quad \operatorname{PP}(\mathrm{k})=1-\operatorname{Pr}(\mathrm{k}, \mathrm{k}+1)$

271 else

$272 P P(k)=$ cdfnormal $(\mathrm{t}, \mathrm{d}, \mathrm{D})-\operatorname{cdfnormal}(\mathrm{s}(\mathbf{f}), \mathrm{d}, \mathrm{D})$

$273 \quad \operatorname{Pr}(\mathrm{k}, \mathrm{k}+1)=1-\mathrm{PP}(\mathrm{k})$

274 end

$275 \mathrm{k}=\mathrm{k}+1$

276 end

$277 \operatorname{Pr}=[\mathrm{Pr}, \mathrm{PP}]$

$278 \operatorname{Pr}=[\operatorname{Pr} ; z e r o s(\operatorname{Pr}(1,:))]$

$279 \operatorname{Dim}=\operatorname{size}(\operatorname{Pr})$

$280 \operatorname{Pr}(\operatorname{Dim}(1), \operatorname{Dim}(2))=1$

$281 \quad \mathbf{y}=\operatorname{Pr}$

282 endfunction

283 function $\mathbf{T r} \_$Mat $=\underline{\mathrm{TM}}(\mathbf{S F})$

284 Tr_Mat $=[]$; 
285 for $\mathrm{f}=\mathbf{S F}$

286 Tr_Mat $=\left[\mathbf{T r} \_M a t, \underline{\mathrm{TM}}(\mathrm{f})\right]$;

287 end

288 endfunction

289

290

291

292

293

294

295

296

297

298

299

300

301

302

303

304 
305

306

10. File 9: DAI_fire.sci (Dynamic Allocation Index of fire)

307 function $\mathbf{y}=\underline{\mathrm{DAI}}(\mathbf{T M})$

$308 \quad \mathrm{P} 1=[]$

309 Product=[];

310 for $1=1: \mathrm{NS}-1$

$311 \mathrm{P} 1=[\mathrm{P} 1, \mathbf{T M}(1,1+1)]$

312 Product $=\left[\right.$ Product, $\left.(\text { alpha^ })^{*} \operatorname{prod}(\mathrm{P} 1) *\left(1+(\text { alpha/ }(1-\text { alpha }))^{*} \mathbf{T M}(1+1, \mathrm{NS}+1)\right)\right]$

313 end

$314 \mathrm{H}=1+(($ alpha*TM$(1, N S+1)) /(1-$ alpha $))+$ sum(Product $)$

315 if $\mathrm{CTLSF}==0$ then

$316 \quad \mathbf{y}=\mathrm{V}(\mathrm{f}) /\left(1-\left(\right.\right.$ alpha $\left.\left.^{\wedge}(\mathrm{T} 0(\mathrm{f})+\mathrm{r}(\mathrm{f}))\right) *(1-(1-\mathrm{alpha}))^{*} \mathrm{H}\right)$

317 else

$318 \quad \mathbf{y}=\mathrm{V}(\mathrm{f}) /\left(1-\left(\text { alpha }{ }^{\wedge}(\mathrm{T}(\mathrm{LSF}, \mathrm{f})+\mathrm{r}(\mathrm{f}))\right)^{*}(1-(1-\mathrm{alpha})) * \mathrm{H}\right)$

319 end

320 endfunction

321

322

323

324

325 